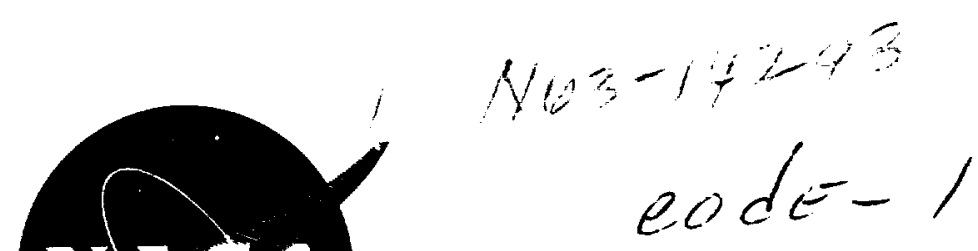

NASA

TECHNICAL NOTE

D-1700

\title{
PROTONS FROM 0.1 TO 5 MEV AND ELECTRONS \\ OF 20 KEV AT 12 EARTH RADII DURING THE SUDDEN \\ COMMENCEMENT ON SEPTEMBER 30, 1961
}

by

R. A. Hoffman, L. R. Davis, and J. M. Williamson

Goddard Space Flight Center

NATIONAL AERONAUTICS AND SPACE ADMINISTRATION WASHINGTON

March 1963 


\title{
PROTONS FROM 0.1 TO 5 MEV AND ELECTRONS OF 20 KEV AT 12 EARTH RADII DURING THE SUDDEN COMMENCEMENT ON SEPTEMBER 30, 1961
}

by

\author{
R. A. Hoffman, L. R. Davis, and J. M. Williamson \\ Goddard Space Flight Center
}

SUMMARY

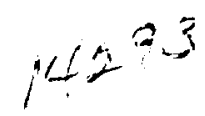

During the period around the sudden commencement of the magnetic storm on September 30,1961, the ion-electron detector aboard Explorer XII observed fluxes of low energy protons and electrons while the satellite was at 12 earth radii and outside the magnetosphere.

About 25 minutes before the sudden commencement, the proton intensity increased; and, 8 minutes before, the omnidirectional intensity suddenly jumped. The maximum flux of about $2 \times 10^{5}$ protons $/ \mathrm{cm}^{2}-\mathrm{sec}$-ster above $140 \mathrm{kev}$ was attained $1 / 2$ hour after the sudden commencement. Thereafter the intensities varied irregularly with time and displayed fluctuations in anisotropy that were dependent on the direction of the local magnetic field. The energy spectrum was independent of both time and intensity. The energy density of the proton spectrum $\left(1 \times 10^{-9}\right.$ $\mathrm{erg} / \mathrm{cm}^{3}$ ) was too small to account for the observed compression of the earth's field on the sunlit side during the magnetic storm.

Time-coincident with the sudden jump in proton intensities, an electron flux first appeared and reached its peak value of $3 \times 10^{6}$ electrons $/ \mathrm{cm}^{2}-\mathrm{sec}-\mathrm{ster}$ in the 10 to $35 \mathrm{kev}$ range about 1 minute before the observance of the sudden commencement on the earth's surface; it continued for about 10 minutes and then disappeared. The detection efficiency for electrons places an upper limit of $2 \times 10^{5}$ electrons $/ \mathrm{cm}-\mathrm{sec}-\mathrm{ster}$ for the remainder of the storm. 



\section{CONTENTS}

Summary $\ldots \ldots \ldots \ldots \ldots \ldots \ldots \ldots \ldots \ldots \ldots$

INTRODUCTION. ...................... 1

INSTRUMENTATION AND MEASUREMENTS . . . . . . 1

THE ORBIT $\ldots \ldots \ldots \ldots \ldots \ldots \ldots \ldots \ldots \ldots \ldots$

GENERAL TIME VARIATIONS. . . . . . . . . . 2

ENERGY $\operatorname{SPECTRA} \ldots \ldots \ldots \ldots \ldots \ldots \ldots$

CONCLUSIONS. .................... 6

ACKNOWLEDGMENT . . . . . . . . . . . 7

References .................... 


\title{
PROTONS FROM 0.1 TO 5 MEV AND ELECTRONS OF 20 KEV AT 12 EARTH RADII DURING THE SUDDEN COMMENCEMENT ON SEPTEMBER 30, 1961*
}

by

\author{
R. A. Hoffman, ${ }^{\dagger}$ L. R. Davis, and J. M. Williamson \\ Goddard Space Flight Center
}

\section{INTRODUCTION}

During the period around the sudden commencement of the magnetic storm on September 30 , 1961, the ion-electron detector aboard Explorer XII (1961 v1) observed fluxes of low energy protons and electrons while the satellite was outside the magnetosphere. This paper presents the results of an initial study of the time and angular variation of these particles.

\section{INSTRUMENTATION AND MEASUREMENTS}

The detector consisted of a thin coating of $\mathrm{ZnS}(\mathrm{Ag})$ deposited on the face of a photomultiplier tube. Particles were collimated to the detector in such a way that the telescope factor was $5.4 \times 10^{-3}$ $\mathrm{cm}^{2}$-ster. Into the collimator was periodically rotated a set of thin nickel absorbers, varying in thickness from an open window to $500 \times 10^{-6} \mathrm{inch}$, in order to obtain range (energy) spectra.

Particle measurements were performed by three modes of operation:

1. Ion Pulse Mode: Proton and heavier ion particle fluxes were measured separately from electrons by eliminating the latter with pulse height discrimination. The maximum energy that electrons could lose in the thin phosphor was small compared with the energy loss by low energy protons. High energy particles (protons $>5 \mathrm{Mev}$ ) were not measured because of their small energy loss in the phosphor.

2. Electron Current Mode: The energy flux of electrons was measured by closing the direct geometry aperture and allowing the incident flux to undergo 90-degree scattering to the phosphor from a gold disk. The dc current from the eighth dynode of the photomultiplier gave a measure of the electron energy flux because the scattering process eliminated protons.

* Presented at The American Geophysical Union Meeting in Washington, April 25-28, 1962. Also published in: J. Geophys. Res. 67(13):502-505, December 1962.

†National Academy of Sciences-NASA Post-Doctoral Resident Research Associate. 
3. Total Current Mode: The energy flux of all incident radiation was measured in direct geometry by telemetering the eighth dynode current. If one type of particle could be eliminated from either mode 1 or 2 , then mode 3 gave an energy flux measurement of the remaining type.

The rotation of successively thicker absorbers into the collimator increased the low energy cutoff for detection. In plotting particle flux energy spectra, the detector has been considered as measuring an integral spectrum above the energy for 50 percent detection efficiency.

In this analysis the measured counting rates and currents are interpreted as consisting of only protons and electrons. However, it should be noted that a second count rate channel, operated similarly to mode 1, was included to determine the fluxes of heavier ions. The results of a preliminary analysis of this channel show that a significant flux of particles heavier than protons existed during the sudden commencement period. These results are dependent on the channel's trigger level, which was set greater than the maximum pulse size from protons, having remained stable. An analysis that will test this requirement is now being made. Should these heavier ions be present, the correction to the proton data would be less than a 20 percent decrease, and the shape of the spectrum would be little affected.

A detailed discussion of the instrumentation is presented in Reference 1.

Since the telescope looked out at a 45-degree angle from the spin direction of the satellite, the detector scanned a cone of 45-degree half-angle with time. Since the sampling rate was about $1 / 3$ second whereas the roll period was 2 seconds, a roll-modulated signal was obtained from nonisotropic fluxes. Because of the particular orientation of the local magnetic field with respect to the spin axis during the sudden commencement period, local pitch angles from 90 degrees down to about 20 degrees were usually sampled.

\section{THE ORBIT}

The satellite was placed into a highly elliptical orbit having an apogee of $83,600 \mathrm{~km}$ on the sunlit side of the earth. With a period of $26-1 / 2$ hours, considerable time was spent beyond the earth's magnetosphere.

For the period covered by the present discussion, both the sun and the earth were essentially in the orbital plane. During the sudden commencement period, the sun longitude was 130 degrees and the earth longitude was -103 degrees, both behind the spin direction, so that the look-cone was effectively away from both bodies.

\section{GENERAL TIME VARIATIONS}

Figure 1 shows the general time variation of protons and electrons around the time of the sudden commencement, which occurred at 2109 UT, September 30,1961 . The upper part of the figure contains the maximum and minimum counting rates of the detector for protons due to the roll modulation 


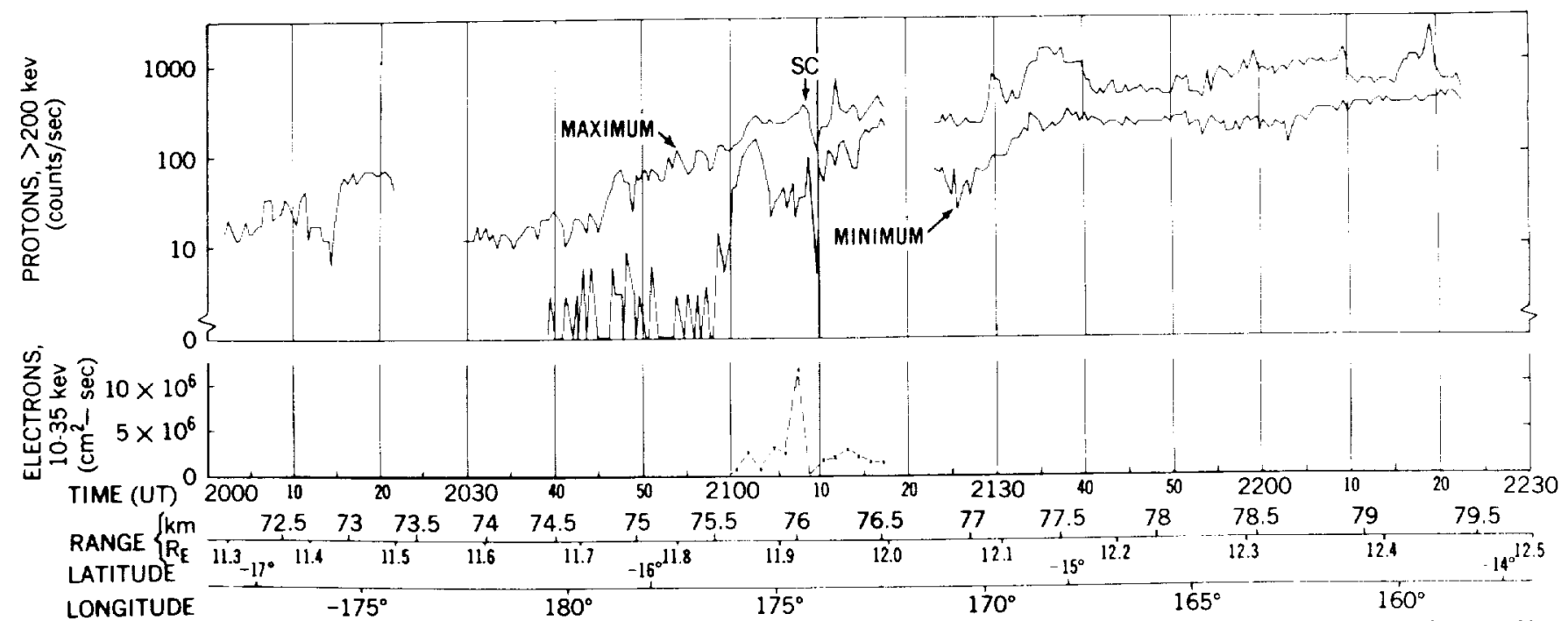

Figure 1 - General time variation of protons and electrons observed by the ion-electron detector aboard Explorer XII during the period around the sudden commencement (SC) of the September 30, 1961, magnetic storm.

for the energy range above $200 \mathrm{kev}$. The difference between the two rates is an indication of the anisotropy of the radiation. During the period 2140 to 2150 the radiation can be considered as $i s o-$ tropic, as will be demonstrated later. During the period 2200 to 2210 the radiation was anisotropic.

The lower part of the figure contains the electron observations in the 10 to $35 \mathrm{kev}$ range and indicates the flux averaged over the roll period and then assumed isotropic over $4 \pi$. The average energy of the electrons was assumed to be $20 \mathrm{kev}$ in order to obtain particle flux estimates from the energy flux, which was actually measured in the scatter geometry (mode 2).

In Figure 1 the following two features are to be noticed:

1. The first involves the period just preceding the time of the sudden commencement on the earth's surface. From about 1900 hours, when the satellite emerged from the outer edge of the geomagnetic field, up until 2045 the radiation consisted primarily of penetrating protons remaining from the September 28 solar proton event (Reference 2). These particles had sufficient energy to reach the phosphor by penetrating the sides of the detector.

Then about 2045 the low energy protons initiated a slow increase until 2100 , when the omnidirectional intensity suddenly jumped-as evidenced by the factor of 100 increase in the minimum counting rate.

Time-coincident with the sudden jump, an electron flux first appeared and reached its peak directional value of $3 \times 10^{6}$ electrons $/ \mathrm{cm}^{2}-\mathrm{sec}$-ster about 1 minute before the sudden commencement. Immediately coincident with the sudden commencement, the electrons momentarily disappeared and the proton intensity dropped. The electrons reappeared and continued until loss of telemetry at 2118 , after which period no electrons were detected. The detection efficiency for electrons places an upper limit of $2 \times 10^{5}$ electrons $/ \mathrm{cm}^{2}$-sec-ster for the remainder of the storm. 

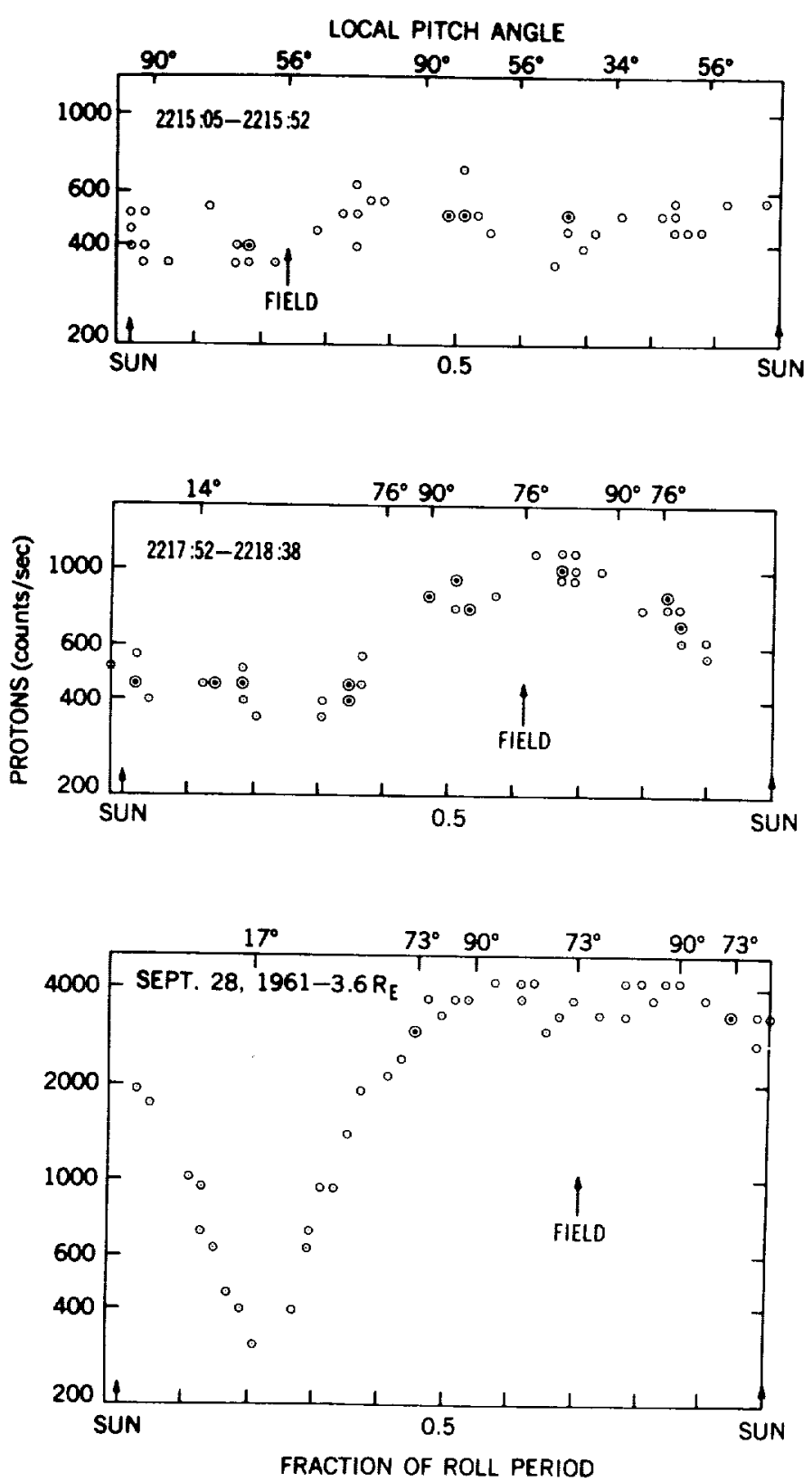

Figure 2 - Roll modulation of detector rates before the burst of radiation at 2218 UT, ir,dicating isotropy over the look-cone of the detector (top); the anisotropy during the burst (middle); and, for comparison with the burst case, the radiation distribution of geomagnetically trapped protons for the same magnetic field angles as the burst (bottom). (Solid circles indicate two data points.)

direction, which has changed since just before the burst. counting rates were similar to pre-burst rates.
The noteworthy feature presented here is the sudden increase in proton intensity and the appearance of electrons preceding in time by 8 or 9 minutes the observation of the sudden commencement on the earth's surface. If this feature is interpreted as the storm front, the time interval is in the same range as that mentioned for hydromagnetic wave propagation [calculated from ray theory (e.g., Reference 3)] from the outer edge of the magnetosphere to the earth's surface.

2. The second feature from Figure 1 concerns the fluctuations in anisotropy of the protons. We notice that during the period beyond 2130 the minimum rate varied rather smoothly while the maximum rate displayed bursts, indicating an anisotropy in the radiation. The nature of the anisotropy is shown in Figure 2, with the burst at 2218 used as a typical event.

The top of the figure contains the roll modulation of the detector rates for several cycles between "see sun" times, as observed by the aspect indicator aboard the satellite, for about seven revolutions just before the burst. No peak in counting rate was observed, so this distribution has been called isotropic. The top of each figure contains the local pitch angle of the particles measured by the detector at a given time, which is derived from a knowledge of the angles of the magnetic field with respect to the satellite spin axis.

The middle plot contains data during the burst and exhibits an increased intensity centered around the spin-field plane From "see sun time" to 0.3 of a roll the

-Obtained through the courtesy of Dr. Laurence Cahill. 
It is important to notice that the superimposed particles were not trapped in the field with the same characteristics as geomagnetically trapped particles. To illustrate this point, the lower part of Figure 2 contains the roll distribution of protons trapped in the geomagnetic field for almost identically the same field direction with respect to the satellite spin axis. We observe in this case a slight minimum intensity in the spin-field plane surrounded by two maxima approximately in the $90-$ degree pitch angle direction. The 73-degree local pitch angle was scanned three times in each roll, with the same counting rate occurring each time.

In the burst case the 76-degree pitch angle was scanned three times, but the rate at the angle occurring in the spin-field plane was considerably higher than the rates at the angle on either side of the 90-degree observations; this means that the particles apparently were arriving predominantly from one hemisphere and were not being mirrored back up the lines of force-the effect is displayed in Figure 3. It appears that the directional change in the local field between 2215 and 2218 acted like a gate, and allowed an increased flux to arrive at the satellite from a preferred direction. These variations are time, not spatial, effects because the satellite was moving only about $1 \mathrm{~km} / \mathrm{sec}$ during this time near apogee.

\section{ENERGY SPECTRA}

The shape of the energy spectrum of these sudden commencement protons appears to be independent of time and direction. Figure 4 contains approximate integral spectra for the period of isotropy before the burst shown in Figure 3, for the peak

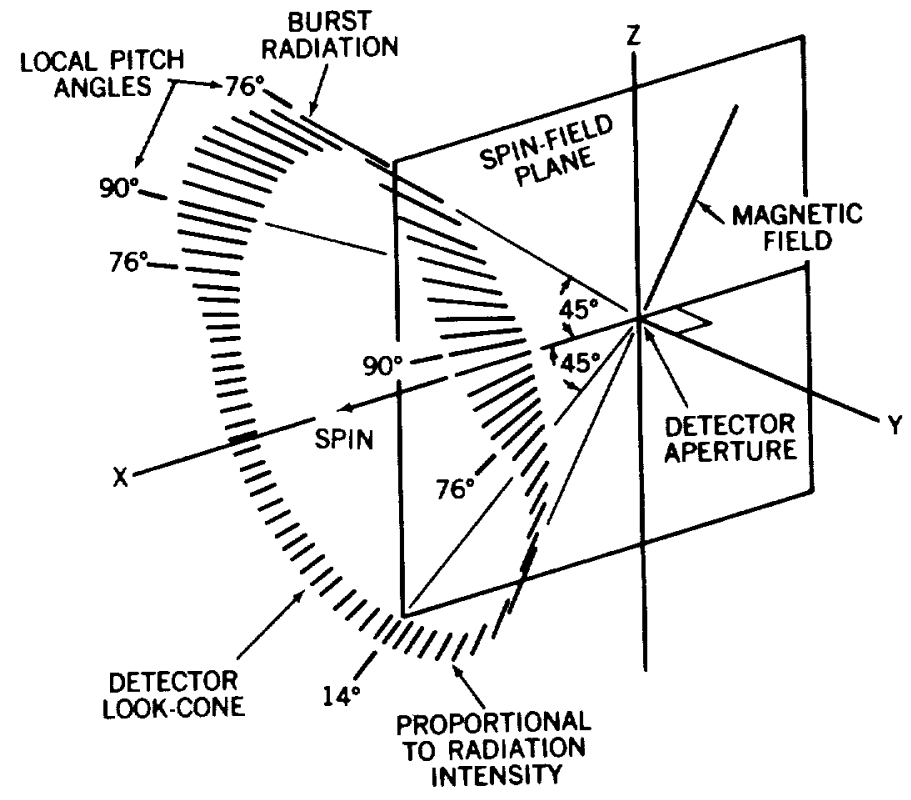

Figure 3 - The geometry of the anisotropy.

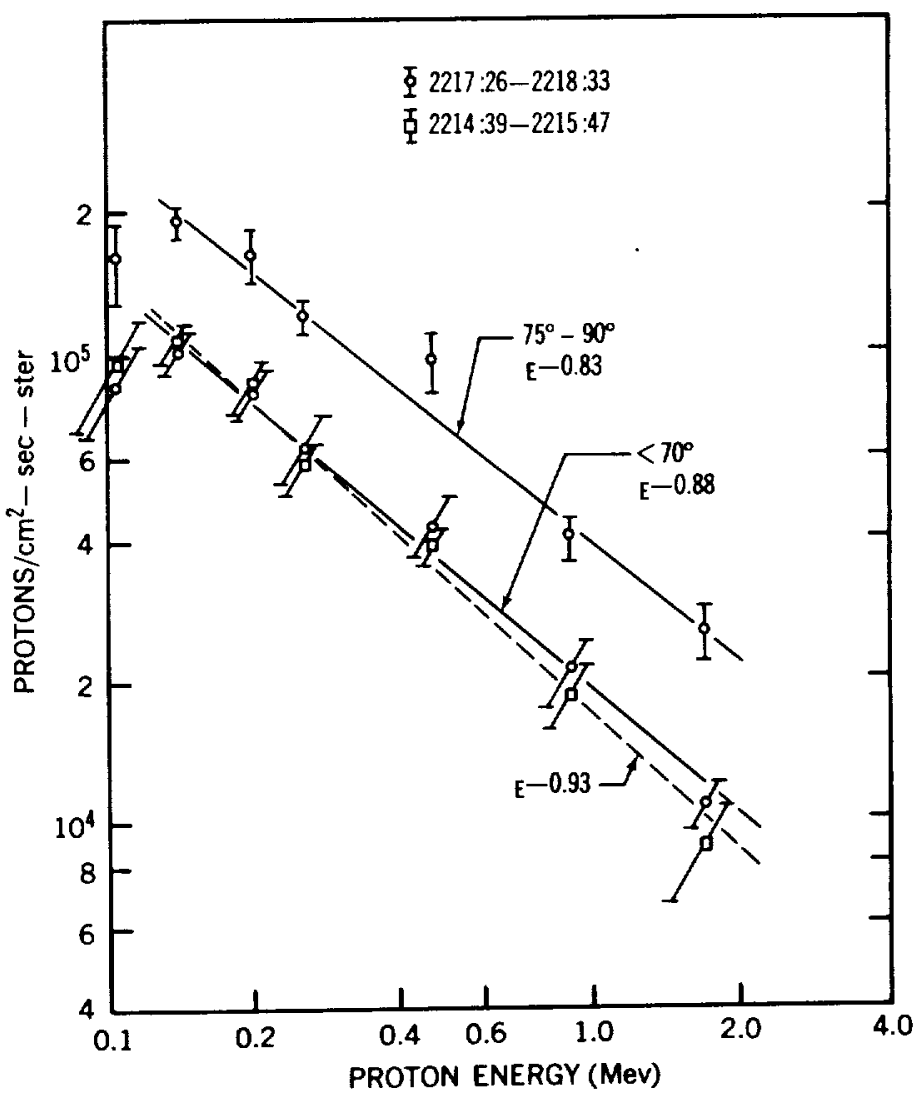

Figure 4 - Integral proton spectra of the radiation during the period of isotropy before the 2218 UT burst and during the burst. 
of the burst (pitch angles 75 to 90 degrees), and for the directions that did not change intensity during the burst $(<70$ degrees).

Numerous spectra have been deduced from data collected during other periods in the time interval 2102 to 0322 . An approximate fit of an assumed power law spectrum, $N(>E)=N_{0} E^{-\gamma}$, to the data points yields a variation in $y$ only from 0.80 to 1.05 .

It would be desirable to extend the energy spectrum to lower energies than the $105 \mathrm{kev}$ level of the lowest energy step plotted in Figure 4. An indication of the lower energy intensities can be deduced from studying the direct geometry dc current (mode 3), which measured the energy flux of the protons after the sudden commencement, because the electron contribution has been eliminated by the scatter geometry measurements (mode 2). The proton energy flux measurements extend to lower energies than the pulse measurements because there is no restriction on pulse size. For the open window in the absorber wheel, the dc current efficiency has a flat maximum from 500 to $1000 \mathrm{kev}$ and very slowly falls off towards lower energy, reaching 50 percent efficiency at $55 \mathrm{kev}$ and 10 percent efficiency at $20 \mathrm{kev}$. The next thicker absorber $\left(5 \times 10^{-6}\right.$ inch $\left.\mathrm{Ni}\right)$ attains 50 percent efficiency at $110 \mathrm{kev}$ and 10 percent at $55 \mathrm{kev}$.

The low energy current channels did not display a significant enhancement of current over the higher energy channels during the sudden commencement period; this indicated a considerable flattening of the integral particle spectrum at energies below $140 \mathrm{kev}$.

The energy density of such a proton spectrum deficient of particles below $140 \mathrm{kev}$ is about $1 \times 10^{-9} \mathrm{erg} / \mathrm{cm}^{3}$. Such an energy density, though significant, is too small to account for the observed compression of the earth's field on the sunlit side during the magnetic storm. If the field strength at the outer edge of the geomagnetic field is considered to be twice the dipole strength because of the compression, the proton energy density could compress the field only to about 16 earth radii. The outer edge of the field, assuming it is characterized by the appearance of trapped radiation, was measured by the detector on the inbound portion of the pass after the sudden commencement at less than 10 earth radii. The energy density at a boundary of this range is $1.6 \times 10^{-8}$ $\mathrm{erg} / \mathrm{cm}^{3}$.

\section{CONCLUSIONS}

The ion-electron detector aboard Explorer XII has shown the existence of a large flux of protons in the 100's-of-kev energy range in a solar plasma. The protons displayed a sudden enhancement preceding by about 8 minutes the time of the sudden commencement on the earth's surface. At the time of the enhancement, electrons from 10 to $35 \mathrm{kev}$ appeared and remained only until a few minutes after the sudden commencement.

The variation in proton intensities after the sudden commencement was dependent on the direction of the local magnetic field but did not indicate that these protons were trapped in the field with the same characteristics as geomagnetically trapped radiation. Rather, the directional changes of the local magnetic field acted like a gate, allowing an increased flux to arrive at the satellite from a preferred direction. 
The integral energy spectrum was independent of both time and intensity. The energy density of the observed proton spectrum, though significant, was too small to account for the compression of the earth's field on the sunlit side during the magnetic storm.

\section{ACKNOWLEDGMENT}

The authors are grateful to Dr. Laurence J. Cahill, Jr., of the University of New Hampshire, for cooperating in the exchange of magnetic field aspect data.

\section{REFERENCES}

1. Davis, L. R., and Williamson, J. M., "Low-Energy Trapped Protons," NASA Technical Note D-1669, 1962.

2. Bryant, D. A., Cline, T. L., et al., "Cosmic Ray Observations in Space," NASA Technical Note D-1669, 1962. A more complete version appears under the title "Explorer XII Observations of Solar Cosmic Rays and Energetic Storm Particles Following the Solar Flare of 28 September 1961," J. Geophys. Research, December 1962.

3. Dessler, A. J., Francis, W. E., and Parker, E. N., "Geomagnetic Storm Sudden-Commencement Rise Times," J. Geophys. Research, 65(9):2715-2719, September 1960. 